\title{
Determinación de plomo en leche materna de madres lactantes en nueve distritos de la ciudad de Lima, Perú
}

\section{Determination of lead levels in breast milk of breastfeeding mothers in nine districts in Lima, Peru}

Correspondencia Daniel Guillen-Mendoza danielkenn@gmail.com

Recibido: 18/01/2017

Arbitrado por pares

Aprobado: 17/05/2017

Citar como: Guillen-Mendoza D, Bellomo S, Torres N, Lazo E, Guillen-Pinto D. Determinación de plomo en leche materna de madres lactantes que viven en el norte de la ciudad de Lima. Acta Med Peru. 2017;34(2):90-4

\author{
Daniel Guillen-Mendoza ${ }^{1, a}$, Sicilia Bellomo ${ }^{1,2, b}$, Nancy Torres, Edwin Lazo ${ }^{1,2, c}$, \\ Daniel Guillen-Pinto ${ }^{1,2, c, d}$ \\ 1 Facultad de Medicina Alberto Hurtado, Universidad Peruana Cayetano Heredia. Lima, Perú. \\ 2 Hospital Nacional Cayetano Heredia. Lima, Perú. \\ 3 Hospital Nacional Carlos Alberto Seguín Escobedo. Lima, Perú. \\ a Médico Cirujano; b Médico Pediatra; c Médico Neurólogo Pediatra; d Doctor en Medicina
}

\section{RESUMEN}

Objetivo: Determinar los niveles de plomo en leche materna en puérperas primíparas provenientes de nueve distritos de la ciudad de Lima. Materiales y método: Se realizó un estudio transversal entre octubre de 2010 y agosto de 2012. Se incluyeron 100 muestras de leche materna, de mujeres que vivieron como mínimo cinco años en la misma zona de Lima. El método de análisis fue la espectrofotometría de absorción atómica. Resultados: El 37\% de las muestras tuvieron un nivel detectable de plomo, seis de ellos entre 5,0 y 9,9 ng/g y cinco mayores de $10 \mathrm{ng} / \mathrm{g}$. No se identificaron condiciones de riesgo asociados. Conclusión: Se concluye que un porcentaje importante de nuestras muestras de leche materna presentaron contaminación con plomo, particularmente en residentes de la zona norte de Lima.

Palabras clave: Plomo; Leche materna; Lactancia materna (fuente: DeCS BIREME).

\section{ABSTRACT}

Objective: To determine lead levels in breast milk in primiparous women from nine districts in Lima. Materials and methods: We conducted a cross-sectional study between October 2010 and August 2012. One-hundred samples of breast milk were included, these were from women who lived for more than 5 years in the same area in Lima. We used the atomic absorption spectrophotometry method for the measurements. Result: Thirty-seven percent of the samples had detectable lead levels, six of them were between 5.0 and $9.9 \mathrm{ng} / \mathrm{g}$ and five were over $10 \mathrm{ng} / \mathrm{g}$. We did not identify associated risk factors. Conclusion: An important percentage of samples of breast milk are contaminated with lead, particularly in persons living in northern Lima.

Keywords: Lead; Milk, human; Breast feeding (source: MeSH NLM). 


\section{INTRODUCCIÓN}

La intoxicación plúmbica es uno de los grandes problemas que afecta al niño en desarrollo. Cada vez existe mayor evidencia acerca de los mecanismos de daño cerebral y su influencia en la salud ${ }^{[1-5]}$. Sin embargo, la mayoría de estudios se enfocan en niños con factores de riesgo ya conocidos, como vivir en comunidades mineras $^{[1,5]}$, en zonas aledañas a depósitos portuarios o en zonas cercanas a desechos industriales ${ }^{[2]}$, mas no se han estudiado los riesgos por exposición crónica en las grandes ciudades ${ }^{[1,2,6]}$.

En la ciudad de Lima, como resultado de varios factores, se presume un incremento de la concentración de plomo en el aire y suelo, principalmente en las zonas urbanas e industriales ${ }^{[4,7,8]}$. Los factores serían el crecimiento desordenado del parque automotor, la existencia de talleres mecánicos o industriales, unidades automotrices en mal estado, fundiciones cercanas a la ciudad, quema de basura no seleccionada en la ciudad (contenido de metales), reciclaje artesanal de baterías y suelos contaminados $^{[1-3,8]}$.

El plomo puede encontrarse en diferentes tejidos y fluidos humanos; se acumula principalmente en los huesos ya que se intercambia por calcio ${ }^{[9]}$. Hacia los años ochenta se encontró que el plomo podía pasar de la madre al bebé por vía transplacentaria y actualmente se conoce mejor la correlación entre los niveles de plomo en sangre materna y sangre fetal ${ }^{[9,10]}$. En años más recientes se encontró plomo en leche materna ${ }^{[11,12]}$. Al respecto, Gulson et al. demostraron una movilización mayor, creciente y sostenida, del plomo esquelético materno durante la lactancia en comparación con el periodo de gestación ${ }^{[13,14]}$. Por su parte, Ettinger encontró que los niveles de plomo en leche materna son influenciados tanto por la exposición ambiental, como por la redistribución del plomo óseo, describiendo una clara correlación entre la plumbemia de la madre, el plomo en la leche materna y la plumbemia en el lactante ${ }^{[15,16]}$.

En nuestro medio no se ha investigado el plomo en leche materna, a pesar que se conoce su presencia en el ambiente. El presente estudio busca determinar las concentraciones de plomo en leche materna de madres primíparas residentes en nueve distritos de la ciudad de Lima, Perú.

\section{MATERIALES Y MÉTODOS}

Estudio observacional y transversal con muestreo no probabilístico. Se incluyeron madres primíparas de neonatos a término, de 20 a 35 años de edad, de 15 a 30 días posparto, que brindaran lactancia materna exclusiva y que vivieran cinco años o más en el mismo sector de la ciudad de Lima. Se excluyó a mujeres con diagnóstico previo de intoxicación por plomo, mujeres con exposición conocida (residencia en puertos y minas) o extranjeras. Se tomaron los datos demográficos y de condiciones de posible riesgo por exposición al plomo ambiental.

La toma de la muestra se realizó en el domicilio de la madre $y$ en instituciones materno-infantiles para las muestras
Tabla 1. Características demográficas de la población estudiada.

\begin{tabular}{lccc} 
Característica & $\begin{array}{c}\text { Plomo no } \\
\text { detectable } \\
\text { (n=63) } \\
\%\end{array}$ & $\begin{array}{c}\text { Plomo } \\
\text { detectable } \\
\text { (n=37) } \\
\%\end{array}$ & $\begin{array}{c}\text { Total } \\
\text { (N=100) } \\
\%\end{array}$ \\
\hline $\begin{array}{l}\text { Edad (años) } \\
\text { Media }\end{array}$ & 23,28 & 24,54 & 23,71 \\
Grado de instrucción & & & \\
Primaria & $9,5 \%$ & $10,8 \%$ & $10,0 \%$ \\
Secundaria & $55,6 \%$ & $59,5 \%$ & $55,0 \%$ \\
Universitaria & $34,9 \%$ & $29,7 \%$ & $35,0 \%$ \\
Ocupación & & & \\
Ama de casa & $47,6 \%$ & $56,8 \%$ & $51,0 \%$ \\
Estudiante & $12,7 \%$ & $5,4 \%$ & $10,0 \%$ \\
Empleada & $39,7 \%$ & $37,8 \%$ & $39,0 \%$ \\
Zona de residencia & & & \\
Norte de Lima* & $84,1 \%$ & $97,3 \%$ & $89,0 \%$ \\
Resto de Lima† & $15,9 \%$ & $2,7 \%$ & $11,0 \%$ \\
\hline
\end{tabular}

* Incluye los distritos de San Martín de Porres, Los Olivos, Independencia, Comas, Carabayllo y Puente Piedra.

+ Incluye los distritos de San Borja, Surco y La Molina.

correspondientes a madres residentes en distritos del norte de Lima (San Borja, Surco y La Molina). Se trató en todo momento de buscar ambientes estériles; sin embargo, en los domicilios, no se pudo garantizar esta condición. Asimismo, las muestras de madres provenientes del norte de Lima (San Martín de Porres, Los Olivos, Independencia, Comas, Carabayllo y Puente Piedra) se obtuvieron en un ambiente estéril en el Hospital Nacional Cayetano Heredia (HNCH). Todos los contenedores estuvieron sellados hasta el momento de la toma de muestra usando guantes estériles. Previamente, se lavó la mama con agua de mesa no gasificada o bidestilada y, la muestra de leche se obtuvo por extracción manual, recolectándose en un contenedor de plástico transparente sin aditivos, con volumen mínimo de $20 \mathrm{~cm}^{3}$; siendo supervisado el proceso por una enfermera. Se siguieron los estándares de recolección y transporte del laboratorio CETOX, laboratorio de referencia en Lima, el cual tiene certificación ISO/ IEC 17025:2006.

La muestra fue transportada en un recipiente con una temperatura entre 4 a $8{ }^{\circ} \mathrm{C}$. El tiempo transcurrido entre la obtención de la muestra y su procesamiento siempre fue menor de 24 horas. El análisis fue realizado por el método de absorción atómica. La concentración fue expresada en $\mathrm{ng} / \mathrm{g}$, equivalentes a $\mathrm{mg} / \mathrm{dL}$. El nivel mínimo de detección fue de 0,97 $\mathrm{ng} / \mathrm{g}$. Una vez obtenidos los resultados, fueron entregados a las madres participantes del estudio, a quienes además se les brindó orientación.

Los datos fueron analizados con el programa SPSS versión 17.0. Para las variables con distribución normal se usó la comparación de medias por la prueba t de Student. Para variables sin 
Tabla 2. Distribución de las características de la población por presencia de plumbemia en 100 muestras de leche materna de madres de diferentes distritos de Lima, Perú.

\begin{tabular}{|c|c|c|c|c|}
\hline Característica & Categoría & $\begin{array}{c}\text { Plomo no } \\
\text { detectable } \\
n(\%)\end{array}$ & $\begin{array}{c}\text { Plomo } \\
\text { detectable } \\
\mathbf{n}(\%)\end{array}$ & $\begin{array}{l}\text { Total } \\
\text { n (\%) }\end{array}$ \\
\hline \multirow[t]{8}{*}{$\begin{array}{l}\text { Factores de riesgo por } \\
\text { cercanía a la residencia }\end{array}$} & Avenidas principales & $13(20,6)$ & $13(35,1)$ & $26(26,0)$ \\
\hline & Vivienda de más de 40 años & $4(6,3)$ & $6(16,2)$ & $10(10,0)$ \\
\hline & Fábricas & $13(20,6)$ & $6(16,2)$ & $19(19,0)$ \\
\hline & Ferreterías/Productos de construcción & $2(3,2)$ & $0(0,0)$ & $2(2,0)$ \\
\hline & Grifos & $5(7,9)$ & $5(13,5)$ & $10(10,0)$ \\
\hline & Uso de plaguicidas & $0(0,0)$ & $2(5,4)$ & $2(2,0)$ \\
\hline & Taller automotriz & $9(14,3)$ & $6(16,2)$ & $15(15,0)$ \\
\hline & Paradero de mototaxis & $0(0,0)$ & $2(5,4)$ & $2(2,0)$ \\
\hline \multirow{8}{*}{$\begin{array}{l}\text { Factores de riesgo por } \\
\text { cercanía del centro laboral } \\
\text { o educativo }\end{array}$} & Avenidas principales & $40(63,5)$ & $18(48,6)$ & $58(58,0)$ \\
\hline & Edificio de más de 40 años & $0(0,0)$ & $1(2,7)$ & $1(1,0)$ \\
\hline & Fábricas & $16(25,4)$ & $12(32,4)$ & $28(28,0)$ \\
\hline & Ferreterías/Productos de construcción & $2(3,2)$ & $5(13,5)$ & $7(7,0)$ \\
\hline & Grifos & $17(27,0)$ & $5(13,5)$ & $22(22,0)$ \\
\hline & Uso de plaguicidas & $0(0,0)$ & $0(0,0)$ & $0(0,0)$ \\
\hline & Taller automotriz & $4(6,3)$ & $2(5,4)$ & $6(6,0)$ \\
\hline & Paradero de mototaxis & $1(1,6)$ & $1(2,7)$ & $2(2,0)$ \\
\hline
\end{tabular}

Mototaxis: motocicleta de tres ruedas y con techo que se usa como medio de transporte público.

distribución normal se utilizó la prueba de Wilcoxon. Se usaron regresiones logísticas en la comparación de muestras con niveles tóxicos y sin ellos.

El proyecto de investigación fue aprobado por el comité de ética de la Universidad Peruana Cayetano Heredia

\section{RESULTADOS}

Se analizaron 100 muestras de leche materna, de las cuales $37 \%$ tuvieron niveles detectables de plomo. Las muestras fueron tomadas entre setiembre del 2010 y agosto del 2012. La mediana de la edad materna fue de 23 años y la media de 23,7 . La edad gestacional promedio fue 39,1 semanas. El $89 \%$ de muestras pertenecieron a madres del norte de Lima. No se encontró correlación de la concentración de plomo con la edad materna, el grado de instrucción ni con la ocupación. Los datos demográficos de la muestra se describen en la Tabla 1.

En cinco muestras se encontraron niveles mayores a $10 \mathrm{ng} / \mathrm{g}$, con una concentración máxima de 30,2 ng/g. En el rango de 5,0 a $9,9 \mathrm{ng} / \mathrm{g}$ se encontraron seis muestras. Siete de las 11 muestras con concentración mayor a 5,0 ng/g correspondieron a madres procedentes de los distritos de San Martín de Porres y Los Olivos.

El mayor número de muestras con niveles detectables fueron de madres provenientes de los distritos de San Martín de Porres,
Los Olivos, Independencia y Comas (Figura 1). Comparando las medias de la concentración no se encontró diferencia entre los niveles de plomo de las puérperas de acuerdo con la edad materna, tiempo de residencia, ni condiciones de riesgo ambientales asociadas a lugar de residencia.

No se encontró asociación entre el tiempo de residencia en un distrito y los niveles detectables de plomo. En conjunto, la población procedente de los distritos del norte de Lima tuvo mayor frecuencia de niveles detectables 40,4\% (36/89), comparada con el resto de distritos $9,2 \%(1 / 11)(p \leq 0,05)$. No hubo diferencia de exposición en las condiciones de riesgo ambiental por zona de residencia entre los grupos de madres con niveles de plomo detectable y no detectable. Asimismo, no se encontró diferencia de acuerdo con las condiciones de riesgo por cercanía al centro laboral o académico entre ambos grupos (Tabla 2).

\section{DISCUSIÓN}

El plomo es un metal pesado sin ninguna función en el cuerpo humano. Desde hace cientos de años se han reportado casos severos de intoxicación con niveles altos de plumbemia. Sin embargo, en la actualidad se conoce que "niveles bajos" también pueden generar serios problemas en el sistema nervioso en desarrollo ${ }^{[17-19]}$, por daño directo de la oligodendroglia inmadura, organización y mielinización axonal; siendo particularmente vulnerable la población de fetos y neonatos ${ }^{[20]}$. 


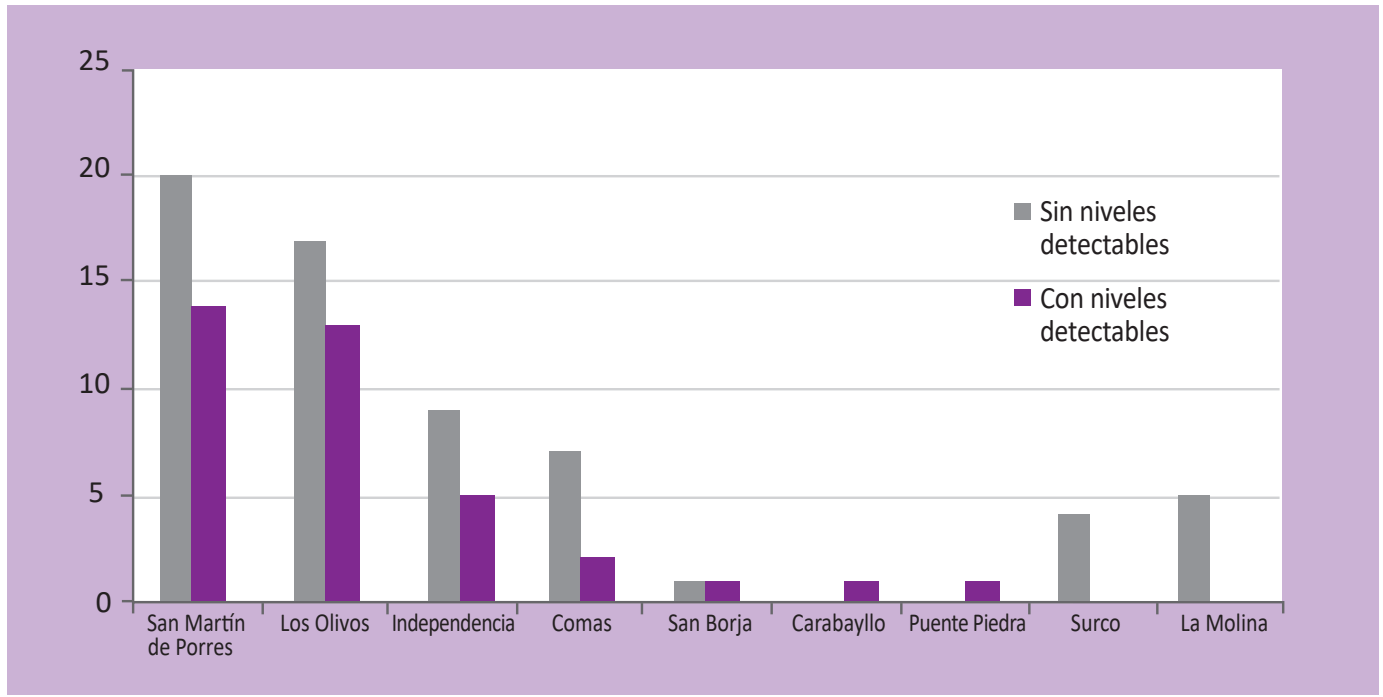

Figura 1. Número de muestras con niveles detectables de plomo por distrito de residencia en Lima, Perú.

El plomo afecta la inteligencia, atención, balance, velocidad de reacción y conducta. Incluso si los niveles fueran detectables sólo en el nacimiento y luego se corrigieran ${ }^{[17-19]}$. Al respecto se ha observado que la plumbemia en la infancia estaría asociada con un decremento en el volumen cerebral, particularmente en la sustancia gris de las regiones frontales ${ }^{[21,22]}$.

Las vías de contaminación plúmbica son oral, inhalatoria, transplacentaria o dérmica. Existe una correlación entre las concentraciones de plomo en leche materna con niveles de plumbemia en los recién nacidos y sus madres. Esto demuestra que la leche materna podría ser una fuente importante de contaminación para el neonato. Se conoce también de una correlación entre la presencia de plomo en leche materna y plumbemia en el recién nacido; sin embargo, no se cuenta con estudios que hayan determinado la proporción exacta de plomo absorbido por lactancia ni una razón de proporciones entre la concentración en sangre y leche materna. De acuerdo con publicaciones recientes la razón de leche a plasma parece ser más alta de lo que antes se esperaba ${ }^{[16]}$.

En nuestro estudio observamos que la mayoría de muestras y los niveles más altos correspondieron a mujeres procedentes de la zona norte de Lima, probablemente por la accesibilidad a la toma de muestra. Pese a ello, debemos denotar que todas las muestras con niveles con niveles detectables, proporcionalmente estas fueron mucho más frecuentes en los distritos del norte de Lima y sólo hubo una muestra correspondiente a otras zonas. Las proporciones más altas se observaron, especialmente, en los distritos de San Martín de Porres y Los Olivos.

El norte de Lima se caracteriza por menor urbanismo, con áreas de suelo expuesto, pocas áreas verdes, zonas industriales, desechos y talleres aledaños al área urbana. Probablemente el menor tamaño muestral y falta de inclusión de otras zonas con urbanismo semejante no permitieron identificar los factores de riesgo asociados. Sin embargo, la fortaleza del presente estudio ha sido demostrar la existencia de contaminación plúmbica de leche materna. En la misma zona norte de Lima, se ha reportado una población materno-infantil en la que se encontró 30\% con niveles mayores de $5 \mu \mathrm{g} / \mathrm{dL}$ en sangre de cordón umbilical; siendo el tiempo de residencia el principal factor asociado ${ }^{[7]}$. Para aclarar estos hallazgos y los actuales proponemos hacer un estudio poblacional de plumbemia materna, neonatal y leche materna como el realizado por Ettinger et al. ${ }^{[16]}$.

En otras ciudades, como Teherán en Irán, la contaminación por plomo es mucho más grave y se encuentran niveles de hasta $23,66 \pm 22,43 \mu \mathrm{g} / \mathrm{L}$ en leche materna. Siendo este uno de los promedios más altos reportados en la literatura ${ }^{[23]}$. Aun así, no se ha determinado propiamente los niveles tóxicos para leche materna, pero se reconoce que cualquier nivel de plumbemia es tóxico incluso cuando los neonatos están asintomáticos ${ }^{[24-26]}$.

Las limitaciones del estudio incluyen el haber realizado un muestreo por conveniencia. El tamaño de muestra limitado no permite extrapolar los resultados a la población; sin embargo, a la luz de los resultados, se denota la necesidad de plantear futuras investigaciones.

En conclusión, dentro de la muestra estudiada, se ha encontrado una cantidad importante de madres con leche materna contaminada con plomo, esto es particularmente preocupante teniendo en cuenta que se trataba de una población aparentemente no expuesta. Queda a futuro determinar el impacto de esta fuente potencial de contaminación en poblaciones de riesgo.

Fuente de financiamiento: Proyecto financiado por la Beca para Alumnos del Fondo de Investigación de la Facultad de Medicina Alberto Hurtado, de la Universidad Peruana Cayetano Heredia.

Conflictos de interés: Los investigadores declaran no tener conflictos de interés. 


\section{REFERENCIAS BIBLIOGRÁFICAS}

1. Castro-Bedriñana J, Chirinos-Peinado D, Ríos-Ríos E. Niveles de plomo en gestantes y neonatos en la ciudad de la Oroya, Perú. Rev Peru Med Exp Salud Publica. 2013;30(3):393-8.

2. Guo P, Xu X, Huang B, Sun D, Zhang J, Chen X, et al. Blood lead levels and associated factors among children in Guiyu of China: a population-based study. PLoS One. 2014;9(8):e105470.

3. Cao J, Li M, Wang Y, Yu G, Yan C. Environmental lead exposure among preschool children in Shanghai, China: blood lead levels and risk factors. PLoS One. 2014;9(12):e113297.

4. Espinoza R, Hernández-Avila M, Narciso J, Castañaga C, Moscoso $\mathrm{S}$, Ortiz $\mathrm{G}$, et al. Determinación de los niveles de plomo en sangre de niños del Callao y el área de Lima Metropolitana. Salud Pública Mex. 2003;45 supl 2:209-19.

5. Pebe G, Villa H, Escate L, Cervantes G. Niveles de plomo sanguíneo en recién nacidos de La Oroya, 2004-2005. Rev Peru Med Exp Salud Publica. 2008;25(4):355-60

6. Fernández, G. Hernández-Avila, M. Niveles de plomo sanguíneo en madres y recién nacidos derechohabientes del Instituto Mexicano del Seguro Social. Salud Publica de Mex. 2000;42(5):391-6.

7. Guillen-Mendoza D, Escate-Lazo F, Rivera-Abbiati F, Guillén-Pinto D. Plomo en sangre de cordón umbilical de neonatos nacidos en un hospital del norte de Lima. Rev Peru Med Exp Salud Publica. 2013;30(2):224-8.

8. Korc ME. Exposición a contaminantes del aire en áreas urbanas e industriales del Perú. Hojas de Divulgación Técnica. 2004;(94):1-10.

9. Productos químicos y metales. En: Paris E, Rios JC, ed. Intoxicaciones: Epidemiología, clínica y tratamiento. 2da Edición. Santiago de Chile: Ediciones Universidad Católica de Chile; 2005

10. Rivas Padilla F, Vicuña Fernández N, Wong Ramírez S. Exposición urbana no ocupacional al plomo y niveles sanguíneos en mujeres embarazadas y en recién nacidos. Mérida. Venezuela. Rev Fac Nac Salud Pública. 2000;18(2):73-81.

11. Li, PJ. Sheng, YZ. Wang, QY. Gu, LY. Wang, YL. Transfer of lead via placenta and breast milk in human. Biomed Environ Sci. 2000;13(2):85-9.

12. Koyashiki GA, Paoliello MM, Tchounwou PB. Lead levels in human milk and children's health risk: a systematic review. Rev Environ Health. 2010;25(3):243-53.

13. Gulson BL, Jameson CW, Mahaffey KR, Mizon KJ, Patison N, Law $\mathrm{AJ}$, et al. Relationships of lead in breast milk to lead in blood, urine, and diet of the infant and mother. Environ Health Perspect. 1998;106(10):667-74.

14. Gulson BL, Mizon KJ, Palmer JM, Korsch MJ, Taylor AJ, Mahaffey KR. Blood lead changes during pregnancy and postpartum with calcium supplementation. Environ Health Perspect. 2004;112(15):1499-507.

15. Ettinger AS, Téllez-Rojo MM, Amarasiriwardena C, GonzálezCossío T, Peterson KE, Aro A, et al. Levels of lead in breast milk and their relation to maternal blood and bone lead levels at one month postpartum. Environ Health Perspect. 2004;112(8):92631.

16. Ettinger AS, Roy A, Amarasiriwardena CJ, Smith D, Lupoli N, Mercado-García A, et al. Maternal blood, plasma, and breast milk lead: lactational transfer and contribution to infant exposure. Environ Health Perspect. 2014;122(1):87-92.

17. Bhattacharya A, Shukla R, Dietrich KN, Bornschein RL. Effect of early lead exposure on the maturation of children's postural balance: a longitudinal study. Neurotoxicol Teratol. 2006;28(3):376-85.

18. Chiodo LM, Jacobson SW, Jacobson JL. Neurodevelopmental effects of postnatal lead exposure at very low levels. Neurotoxicol Teratol. 2004;26(3):359-71.

19. Lanphear BP, Hornung R, Khoury J, Yolton K, Baghurst $\mathrm{P}$, Bellinger $D C$, et al. Low-level enviromental lead exposure and children's intellectual function: an international pooled analysis. Environ Health Perspect. 2005;113(7):894-9.

20. Zhang J, Peterson SM, Weber GJ, Zhu X, Zheng W, Freeman JL. Freemana. Decreased axonal density and altered expression profiles of axonal guidance genes underlying lead $(\mathrm{Pb})$ neurodevelopmental toxicity at early embryonic stages in the zebrafish. Neurotoxicol Teratol. 2011;33(6):715-20.

21. Cecil KM, Brubaker CJ, Adler CM, Dietrich KN, Altaye M, Egelhoff $\mathrm{JC}$, et al. Decreased brain volume in adults with childhood lead exposure. PLoS Med. 2008;5(5):e112.

22. Brubaker CJ, Dietrich KN, Lanphear BP, Cecil KM. The influence of age of lead exposure on adult gray matter volume. Neurotoxicology. 2010;31(3):259-66.

23. Soleimani S, Shahverdy MR, Mazhari N, Abdi K, Gerayesh Nejad S, Shams $S$, et al. Lead concentration in breast milk of lactating women who were living in Tehran, Iran. Acta Medica Iranica. 2014;52(1):569 .

24. Dorea JG. Mercury and lead during breast-feeding. Br J Nutr. 2004;92(1):21-40.

25. Choi J, Tanaka T, Koren G, Ito S. Lead exposure during breastfeeding. Can Fam Physician. 2008;54(4):515-6.

26. Centers for Disease Control and Prevention. CDC Response to Advisory Committee on Childhood Lead Poisoning Prevention. Recommendations in "Low Level Lead Exposure Harms Children: A Renewed Call of Primary Prevention" [Internet]. Atlanta, GA: CDC; 2012 [citado en 6 de julio de 2017]. Disponible en: https://www. cdc.gov/nceh/lead/acclpp/cdc_response_lead_exposure_recs. pdf 